\title{
LTE User Association for Self-Deployable Networks in Disaster Management Scenarios
}

\author{
Romain Pujol, Razvan Stanica, Fabrice Valois \\ Univ Lyon, INSA Lyon, Inria, CITI \\ Villeurbanne, France \\ firstname.lastname@insa-lyon.fr
}

\begin{abstract}
In a disaster scenario, both victims and first responders need an access to a communication network for applications with different quality of service requirements (e.g. short delay, high throughput). As LTE compatible devices are very common nowadays, LTE shows a lot of advantages to be used as a disaster management network. However, its already deployed infrastructure may be damaged or unusable following the said disaster. In this scenario, self-deployable networks shine as they are quickly deployable and can cover areas inaccessible to humans. In this paper, we present self-deployable networks and show what the 3GPP LTE standard provides to them. We focus on the legacy best-SINR based mobile association between user equipment and base stations. Then, we show that such association is agnostic of the quality of service requirements and therefore not suitable to support efficiently the dedicated applications used in disaster management. We then propose several points of improvement in this association scheme that take specific disaster management constraints into account.
\end{abstract}

Index Terms-user association, self-deployable, disaster management, LTE, private mobile radio

\section{INTRODUCTION}

In a disaster scenario, first responders (e.g. firefighters, paramedics) and victims need a reliable, resilient and secure network to communicate. In order for them to successfully execute their missions or to reveal their presence, they need to efficiently receive voice calls, transmit videos or send data. Such applications have different quality of service (QoS) requirements, some of them being delay sensitive and/or needing high throughput. Therefore, the mobile network plays a very important role in disaster management. After several years of work from the Third Generation Partnership Project (3GPP) to improve and specify the LTE standard, and considering that LTE compatible devices are omnipresent, LTE is the perfect candidate to serve as a network technology for public safety and disaster management use.

As a matter of fact, the LTE standard already contains specifications linked to disaster management and public safety [1]. These specifications try to make the current commercial network compatible with the public safety operation needs, but the environment of a disaster often implies that the already deployed cellular network is partially or totally down following the catastrophe. With the advance of technology and the reduction in size of computing elements, self-deployable networks are a very promising solution to bring connectivity to areas with damaged infrastructures or inaccessible to humans and vehicles. Self-deployable networks often consist of a cluster of autonomous aerial or terrestrial vehicles carrying a light-weight base station (BS) co-located with an Evolved Packet Core (EPC) network [2] [3]. This cluster of mobile BS can then bring coverage to devastated areas and build a fully connected LTE network, which can be used both autonomously or in inter-connection with the existing commercial infrastructure, if the latter is still operable.

However, current LTE standardisation does not respond completely to the needs of target applications for selfdeployable networks in disaster management scenarios. Until now, on a legacy LTE network, when users try to establish communication to BSs, the standard best-SINR based user association mechanism is used. Applying this basic mechanism to self deployable networks would restrict the potential of the network. Indeed, this mechanism is agnostic of the QoS requirements of first responder applications and it does not take into account the mobility of the BS, leading then to suboptimal user association, waste of network resources and poor application performance. We believe this classical approach must be improved for the vital applications required in a disaster management context.

In this paper, we introduce original concepts to build new mechanisms for user association applied to self-deployable networks. We review the current user association mechanism, show its limits in disaster situations using self-deployable networks and we propose several improvements for the first responders needs. The paper is organized as follows. Section II provides an overview of public safety solutions in LTE. Section III presents self-deployable networks for disaster management. In Section IV, we detail the current user association mechanism in LTE and explain in Section V why it is not suitable to support efficiently the dedicated applications used in disaster management and propose several points of improvement. We discuss ongoing work and conclude in Section VI.

\section{LTE FOR PUblic SAFETY}

Public safety networks are networks providing communication services to public safety entities such as firefighters, paramedics or police. Historical public safety networks, like TETRA and P.25, are based on the 2G legacy standard and do not support data-intensive services such as video streaming or image sharing. Today, LTE supports high bandwidth, low latency, and high security data services, as well as real-time communication. By the beginning of August 2019, the Global 
Mobile Suppliers Association [4] has listed 304 commercially launched LTE-Advanced networks in 134 countries.

The 3GPP standard already initiated the implementation of a few functions to support public safety operations using the LTE networks. Agreements between first responders and mobile operators exist in order to use the existing LTE architecture as a public safety network for first responders operations. The principal public safety functions implemented in the standardisation are:

1) Emergency Calls: Emergency calls enables user equipments (UEs), with or without Universal Subscriber Identity Module (USIM), to make emergency calls for free using any commercial BS available, even those of another operator than the one the user subscribed to.

2) Public Warning System: It allows to provide emergency text messages to all the UEs in a geographical location, even those not yet authenticated to the network.

3) Isolated E-UTRAN Operation for Public Safety: It allows an isolated BS, not linked to a core network, to enable communications for covered UEs. In IOPS, several functions of the core network are co-located with the BS.

4) Device to Device: It enables two UEs to communicate together without passing by the BS. This mechanism also allows uncovered UE to be relayed to the closest BS.

5) Multimedia Broadcast Multicast Service: It is a point-tomultipoint service to provide broadcast and multicast services to UEs which belong to the same mobile network, through efficient mechanisms in the radio access network and the core network.

6) Group Communication System Enablers for LTE : It gives the possibilities to create, to delete, to join or to leave a group of communication. A user can join several groups and can use different services, such as voice or video, in each group.

7) Mission Critical Push To Talk (MCPTT): It allows to only one member of a group, at a given time, to reserve the communication channel to talk to all the users which are forced to listen. MCPTT allows low latency and resource management for local communications.

\section{Self Deployable Networks}

Traditional cellular networks as LTE have a clear separation between Radio Access Network (RAN) and Core Network $(\mathrm{CN})$. The RAN part of the network consists of multiple BS that provide a link from the UE to the network, and from there to the requested services. Whereas the $\mathrm{CN}$ part consists mostly in an aggregation of functions embedded in servers that assure the interconnection between the BSs. The EPC is responsible to route the traffic from a UE to the right destination, to provide security mechanisms and to manage the mobility of the users moving from one BS to another.

In the legacy LTE network architecture, the backhaul link between the RAN and the $\mathrm{CN}$ is always over-provisioned. However, in self-deployable networks, the connectivity is mainly provided by a wireless connection between aerial and/or terrestrial vehicles. Because of the traffic amount on the user plane and the control plane, the mobility of BS, and the non stable wireless channel, backhaul connectivity represents one of the major challenges in self-deployable networks.

Thanks to recent advancements in network virtualisation, it becomes now feasible to assimilate the BSs and EPC in order to embed them into mobile equipment such as an Unmanned Aerial Vehicle (UAV) or even a backpack. For example, [2] presents a solution of a light-weight EPC. It consists in a complete software refactoring of the classic LTE EPC, where the main functions become software defined network applications, and by replacing most of the security functions with precomputed policy.

Following ideas in literature arguing in favor of bringing the $\mathrm{CN}$ closer to the edge of the network, some research has been done in splitting $\mathrm{CN}$ functions between the different present $\mathrm{BS}$, to counter the emergence of an EPC-BS bottleneck link. For example, in [3], the authors propose to study the placement of the EPC functions between several BS to reduce the traffic generated by the control plane.

\section{BEST-SINR ASSOCIATION}

The best-SINR association is the one used in current cellular networks for decades, including in LTE. Easy to implement, easy to manage, easy to configure, there were few reasons to revisit this network mechanism until now.

When a UE turns on and wants to access the network to use a service, two procedures are triggered. The first one is the association of the user to the best suitable cell. A suitable cell is defined as a cell of the selected or home Public Land Mobile Network (HPLMN) with a sufficient signal over noise ratio level. The second procedure is the authentication, used to verify the identity of the user. At the end of a successful authentication, after all the security operations are executed, a UE is denoted as attached to the network, configured with an IP address allowing it to use network services. The CN permanently keeps in a database the localization of the UE, in order to quickly reach it in case of an input call, or at the arrival of other input data. The procedure to wake up an UE and request it to listen for incoming traffic is known as the paging procedure. The 3GPP TS 24.301 standard [5] provides further details on attachment and paging procedures to interested readers.

\section{A. Technical details and associated procedures}

After powering ON, an UE scans the frequency of the BS at which it was last connected before turning OFF. If no BS of the subscribed HPLMN is found, the UE gets from its own USIM the list of frequencies of its HPLMN. This is known as the Stored Information Cell Selection. If no suitable cell is found on these stored frequencies, the Initial Cell Selection process is started. More details about UE procedures in idle mode can be found in 3GPP TS 36.304 [6]. The following describes the different steps an UE has to follow from powering ON to the Random Access Channel ( $\mathrm{RACH})$ procedure that grants a connection from the selected BS to the UE:

1) Frequency search: The UE scans the stored frequencies or scans all the frequencies allocated to the LTE spectrum. 
2) Time and frame synchronisation: The UE scans each frequency, looking for a typical pattern broadcast by the BS. This allows the UE to synchronise with LTE Ressource Blocks (RB) and to detect the beginning of the LTE frame.

3) MIB decoding: By decoding the previous signals, the UE knows where to find the Master Information Block (MIB), which is broadcast periodically by the BS. This block contains information about the system bandwidth, system frame number and other information helping logical synchronisation.

4) Cell Specific Reference Signal detection: Thanks to the $\mathrm{MIB}$, the UE is able to detect the Cell Specific Reference Signal spread over all the system bandwidth, obtaining an indication of the overall signal quality provided by the BS. For this, the UE measures the Reference Signal Received Power (RSRP) and the Reference Signal Received Quality (RSRQ).

5) SIBs decoding: System Information Blocks (SIBs) are also periodically broadcasted by the BS. They contain various information about the system, such as the PLMN identifier. Several SIB messages exist: SIB1 and SIB2 are mandatory, whereas around 20 other optional SIBs can be transmitted by the operator.

SIB1 contains the PLMN identifier, the BS identifier, the minimum reception power level required to ask for an association, an offset value of reception level to trick the process of cell selection, and a list of all the SIBs broadcasted by the cell. The offset value is used essentially for picocells and femtocells, to allow them to be more easily selected than macrocells when present, for offloading purposes. SIB2 contains radio resources configuration information that is common to all UEs.

Other SIBs can contain information about others BSs, default values for the reselection process in case of an handover, etc. SIB10 and SIB11 contain for example the Earthquake and Tsunami Warning System information, broadcast to all UEs, even those not attached to the network, in case of a disaster.

6) Cell Selection: Once the EU gets the PLMN identifier and the RSRP and RSRQ values, the cell selection begins. To put it in a nutshell, the UE lists the detected cells (from its HPLMN or others PLMN) by order of greater RSRP and RSRQ values. It tries to associate, starting from the cells of its HPLMN until the ones with low RSRP and RSRQ values. And if the association is not successful, it follows the list of others PLMN cells to get access to limited services, such as emergency calls, and to receive Public Warning System messages. 3GPP TS 36.304 [6] details the cell selection procedures.

7) Initial RACH Process: The Random Access Procedure consists of four steps. It is a contention-based procedure to deal with several UEs that try to access the channel at the same time. First, the UE sends on the dedicated Random Access Channel one of the 64 possible RACH preambles. Second, the BS responds with a temporary identifier for the UE and a scheduling grant to send data on the Physical Uplink Shared Channel. Third, all UEs competing for access send back on the granted channel their newly acquired temporary identifier, possibly resulting in a collision. Fourth, if the BS is not able to decode a message (e.g. in case of a collision), the UEs do not receive any response and restart the process from the beginning. If the BS is able to decode one of the UEs message, it responds with an acknowledgement and the UE is then associated.

\section{B. Quality of service support and default bearer}

In cellular networks, even in LTE, bearers define the different classes of quality of service which are supported and offered to UE. Basically, a bearer defines an expected performance in terms of throughput, packet error rate and latency. When a UE associates to the cellular network, whatever is its running application and QoS requirements, a default bearer is allocated. If the current BS is not capable of providing a bearer with the desired quality, a handover is executed, leading to control traffic, delay and increasing association failure. Moreover, when the default bearer is used, it can be over-provisioned regarding to some applications (e.g. text messaging).

\section{ASSOCIATION FOR DISASTER MANAGEMENT SCENARIOS}

We now better understand how the user association works in LTE and we can highlight several drawbacks that make it not suitable for disaster management scenarios.

To sum up the user association in LTE, the UE tries to find the first BS of its HPLMN with acceptable RSRP and RSRQ values. This association is based on the strength of the signal only. This is completely agnostic to the main challenges self-deployable networks are facing: the restricted backhaul capacity and the mobility of both UE and BS. The restricted backhaul capacity between the different BSs can heavily impact the number of rejected user association request, and delay even more the process of cell selection. With BS embedded in UAVs or autonomous vehicles, new challenges appear due to the potential mobility of the BS: if a BS provides the best SINR at a given time, due to its mobility it can become the worst very shortly, leading to a non optimal association.

Moreover, the best-SINR association mechanism presents some cell priority rules, but neither UE nor QoS priority rules. Some applications are more important than others in a disaster management scenario, and some users need higher priority. With the best-SINR association, a UE can be associated to a BS with a strong signal level, but which has not enough capacity to support the user application: an emergency video call from a firefighter may be rejected or delayed until a new association is triggered on a BS which is able to support the traffic. Such situation would appear very often in selfdeployable networks and in public safety scenario. We argue that user association based on metrics such as delay, data rate or priority is needed, especially for disaster management.

Finally, if a group of users need to be associated simultaneously with the same performance objectives (e.g. throughput), the best-SINR association could associate the users to different $\mathrm{BS}$, leading to different performance profiles.

\section{A. Research Challenges}

We claim that, in the case of disaster management, the user association has to be rethought to efficiently support quality 
of service requirements and user priority, even if some related work propose relevant contributions which can be applied. In [7], the authors propose an analytical framework for user association that jointly takes into account both radio access and backhaul capacity. They take into account the uplink and downlink traffic demand, the BS load, the backhaul capacities and the topology. This work proposes theoretical bounds and shows significant gains, but practical distributed mechanisms are still missing. We present below several research directions to evolve the standard user association, in order to improve the UE access to the network in critical situations.

1) Backhaul aware association: The restrained capacity of the backhaul in self-deployable networks is one of the major issues and one of the most studied in the literature. Association can be reimagined in order to minimize the utilisation of the overall backhaul capacity. Such a mechanism would dynamically give priority to BS that still have a sufficient remaining link capacity to reach the core network functions: it will reduce the rejection rate of UEs that would try to connect to the same BS in the actual best-SINR association scheme.

We argue that the RAN should take advantage of the already broadcast SIBs to transmit to the UEs their current remaining backhaul capacity, in order to orient their association decision.

2) QoS aware association: A QoS-aware association could use the existing SIBs as well, to broadcast useful information such as the load of the BS. It could also broadcast information of the neighbor BSs, so that the UE can make the decision of choosing the most suitable BS according to the application requirements.

This association decision could also be made by the network. The UE would give during the $\mathrm{RACH}$ procedure the information of the bearer required for its application, and the RAN could then answer with an association accept or with a detailed rejection, containing a list of close BS, possibly able to grant the requested resources to the UE.

3) Mobility aware association: In self-deployable networks, the mobility of BSs is very beneficial for autonomy and flexibility, but brings some constraints. The limited backchaul is one of them, but the rapidly varying radio link between UE and $\mathrm{BS}$ is another challenge. We saw that the association is based on the strength of the received signal at the UE, and moving the BSs creates a lot of handovers. These handovers increase the control plane traffic and decrease the overall performances of the system.

User association could predict the mobility of both UE and BS to reduce the amount of handovers. From the UE point of view, knowing the destination of a BS could improve the selection process. BS could then broadcast their mobility information thanks to the SIBs. From the BS point of view, the network itself could do a prediction of the best association possible if it was aware of both UEs and BSs mobility. By tracking the devices themselves or by letting them provide their mobility information during the RACH process, the RAN could compute the most suitable BS for each UE.

4) Group association: Group association is a promising feature in disaster scenario. Since teams of first responders often move together, it is less efficient to associate separately UEs that will move the same way and use the same applica- tions. Associating several UEs simultaneously, but separately, consumes resources and takes time. Implementing a common association for a whole group leads to save control traffic and to reduce the association latency. It also guarantees that all the group members are associated to the same BS. In this process, an UE could be responsible to associate the group. Only one UE does the RACH process to avoid contention. This UE should share information about the group (size, applications) to allow the BS to decide the association.

\section{Vi. Concluding Remarks And Future Work}

In this paper, we discussed the user association, especially in the context of a self-deployable network, used in disaster management scenarios. We show that the user-association used in LTE networks has severe drawbacks, mainly because the system information broadcast by the BSs does not announce information about the network capacity or any QoS support.

We propose several improvements for the user association, motivated by the disaster management scenario: backhaul aware association to deal with the topology constraints of selfdeployable network, QoS aware association to allow association to a BS which is able to support the QoS requirement of the user, mobility aware association to deal with both the network and user dynamics, and a group association to do only one association for a group of users, in order to save control traffic and to guarantee that a group will be associated to the same BS.

Our future work will design new SIB messages and add relevant performance indicators to allow a UE to have a better knowledge of the network and the reachable performance, in order to process the best cell selection. Extensions of the 3GPP standards are needed, especially to manage groups of users. We also plan to experiment such new mechanisms on real hardware using Software Defined Radio and software implementation of EPC and BS, such as the SRS LTE software [8], to have a better understanding on what approach could actually function in a real scenario.

\section{REFERENCES}

[1] R. Liebhart, D. Chandramouli, C. Wong, and J. Merkel, LTE for Public Safety. Wiley, 2015.

[2] M. Moradi, K. Sundaresan, E. Chai, S. Rangarajan, and Z. M. Mao, "Skycore: Moving core to the edge for untethered and reliable uav-based lte networks," in Proc. ACM MobiCom 2018, New Delhi, India, Oct. 2018, pp. 35-49.

[3] J. Oueis, V. Conan, D. Lavaux, H. Rivano, R. Stanica, and F. Valois, "Core network function placement in self-deployable mobile networks," Computer Communications, vol. 133, pp. 12-23, Jan. 2019.

[4] (2019) The global mobile suppliers association website. [Online]. Available: https://gsacom.com/

[5] 3GPP, "Non-Access-Stratum (NAS) protocol for Evolved Packet System (EPS); Stage 3," 3rd Generation Partnership Project (3GPP), Technical Specification (TS) 24.301, 2019.

[6] — "Evolved Universal Terrestrial Radio Access (E-UTRA); Use Equipment (UE) procedures in idle mode," 3rd Generation Partnership Project (3GPP), Technical Specification (TS) 36.304, 2019.

[7] N. Sapountzis, T. Spyropoulos, N. Nikaein, and U. Salim, "Optimal downlink and uplink user association in backhaul-limited hetnets," in Proc. IEEE Infocom 2016, San Francisco, CA, USA, Apr. 2016, pp. 1-9.

[8] I. Gomez-Miguelez, A. Garcia-Saavedra, P. D. Sutton, P. Serrano, C. Cano, and D. J. Leith, "srslte: An open-source platform for lte evolution and experimentation," in Proc. ACM WiNTECH 2016, New York, NY, USA, 2016, pp. 25-32. 